\title{
Polinização por beija-flores em uma área de caatinga no Município de Floresta, Pernambuco, Nordeste do Brasil ${ }^{1}$
}

\author{
FABRÍCIA CORREIA LEAL², ARIADNA VALENTINA LOPES ${ }^{3}$ e ISABEL CRISTINA MACHADO ${ }^{3,4}$
}

(recebido: 26 de outubro de 2005; aceito: 20 de julho de 2006)

\begin{abstract}
Pollination by hummingbirds in a "caatinga" area in Pernambuco State, northeastern Brazil). Ornithophilous plants were studied in a caatinga area ( $8^{\circ} 36^{\prime} 00^{\prime \prime}$ S and $38^{\circ} 34^{\prime} 5^{\prime \prime}$ W) in the State of Pernambuco, Northeast of Brazil, from July 2002 to June 2003. Eight ornithophilous species, belonging to five families and seven genera, were registered in the studied community. Cactaceae was the most representative family, with three species pollinated by hummingbirds, followed by Bromeliaceae with two species. Excepting for Bromelia laciniosa Mart. ex Schult. f. and Neoglaziovia variegata (Arruda) Mez (Bromeliaceae), the remaining species had flowering peak in the dry season. Fifty percent of the species have herbaceous habit, followed by shrubs (37.5\%). Most species have red flowers (62.5\%) and all of them have tubular flowers with corolla length of $20.2 \pm 5.6 \mathrm{~mm}$. Sugar concentration in the nectar varied from $18 \%$ to $33 \%$ and the volume from 22 to $41 \mu \mathrm{L}$. There were species flowering throughout the whole year. Five hummingbird species visited the flowers in the community and only one was considered resident. Chlorostilbon aureoventris (d'Orbigny \& Lafresnaye, 1838), due to its frequency and visiting behaviour, was considered as the dominant species. The number of ornithophilous species is lower than those found in other ecosystems as, for example, in the Atlantic forest remnants and some other Neotropical areas. Furthermore, a Trochilinae hummingbird, and not a Phaethornithinae, acted as the "community organizer" species.
\end{abstract}

Key words - caatinga, hummingbirds, northeastern Brazil, ornithophily, pollination

RESUMO - (Polinização por beija-flores em uma área de caatinga no Município de Floresta, Pernambuco, Nordeste do Brasil). Foram estudadas plantas ornitófilas em uma área de caatinga da Reserva Particular do Patrimônio Natural Cantidiano Valgueiro (8³6’00” S e 38³4'5” W) em Pernambuco, Nordeste do Brasil, no período de julho de 2002 a junho de 2003. Oito espécies ornitófilas foram registradas no período de estudo, distribuídas em sete gêneros e cinco famílias. Cactaceae foi a família com maior número de espécies polinizadas por beija-flores, sendo representada por três espécies, seguida de Bromeliaceae, com duas espécies. Foram registradas espécies em floração durante todo o ano. Com exceção de Bromelia laciniosa Mart. ex Schult. f. e Neoglaziovia variegata (Arruda) Mez (Bromeliaceae), todas as demais espécies tiveram pico de floração no período seco. Metade das espécies ornitófilas da comunidade estudada apresentou hábito herbáceo. A maioria das espécies apresentou flores vermelhas (62,5\%), sendo o tipo tubo registrado em todas as espécies. O comprimento médio do tubo da corola foi 20,2 \pm 5,6 mm, a concentração de açúcares no néctar variou de $18 \%$ a 33,5\% e o volume de 22 a $41 \mu \mathrm{L}$. Cinco espécies de beija-flores foram registradas visitando as flores da comunidade estudada, das quais apenas uma foi residente. Chlorostilbon aureoventris (d’Orbigny \& Lafresnaye, 1838), devido ao seu comportamento e freqüência de visita, foi considerada como a espécie dominante da comunidade. Comparações com estudos semelhantes evidenciaram que o número de espécies de plantas ornitófilas que floresceram no período de estudo na Reserva foi expressivamente menor do que o encontrado em estudos em remanescentes de Mata Atlântica e áreas neotropicais em geral. Além disso, uma espécie de Trochilinae, e não de Phaethornithinae, atuou como organizadora da comunidade.

Palavras-chave - beija-flores, caatinga, Nordeste do Brasil, ornitofilia, polinização

\section{Introdução}

As características florais de algumas angiospermas como cores vivas, principalmente vermelhas, abundância

1. Parte da dissertação de mestrado de F.C. Leal, Programa de Pós-Graduação em Biologia Vegetal da Universidade Federal de Pernambuco.

2. Universidade Federal de Pernambuco, Programa de Pós-Graduação em Biologia Vegetal, Rua Prof. Nelson Chaves s/n, 50372-970 Recife, PE, Brasil.

3. Universidade Federal de Pernambuco, Departamento de Botânica, Rua Prof. Nelson Chaves s/n, 50372-970 Recife, PE, Brasil.

4. Autor para correspondência: imachado@ufpe.br de néctar, ausência de odor, corolas tubulosas e nectário distante do estigma e das anteras (Faegri \& Pijl 1979, Endress 1994) estão relacionadas a visitas por beijaflores. Dessa maneira, certas flores estão especializadas em menor ou maior grau para a polinização por estas aves, assim como espécies diferentes de beija-flores também estão adaptadas a determinados tipos de flores (Snow \& Snow 1980).

Os beija-flores são exclusivos das Américas e compõem uma das maiores famílias (Trochilidae) de aves, com cerca de 328 espécies, 78 destas ocorrendo no Brasil (Sick 1997, Schuchmann 1999). Dentre os vertebrados os beija-flores são os principais agentes 
polinizadores (Bawa 1990), desempenhando importante papel na polinização de cerca de $15 \%$ das espécies de angiospermas (Feinsinger 1983).

Os estudos sobre polinização por beija-flores apresentam diversos enfoques e abordagens, dividindose em trabalhos que envolvem uma ou poucas espécies de plantas ou beija-flores e aqueles que abordam a guilda de plantas ornitófilas de um determinado local (Fischer 1996). Dentre os trabalhos que enfocam comunidades de plantas ornitófilas, realizados fora do Brasil, destacam-se os de Snow \& Snow (1972, 1980), Feinsinger (1976), Feinsinger \& Colwell (1978), Brown \& Bowers (1985), Arizmendi \& Ornelas (1990), Diedzioch et al. (2003) e Lasprilla \& Sazima (2004), conduzidos principalmente na América Central e nos Andes. No Brasil, estes estudos em nível de comunidade são escassos e concentrados, predominantemente, na região sudeste (Snow \& Teixeira 1982, Sazima et al. 1995, 1996, Buzato et al. 2000, Vasconcelos \& Lombardi 2000, 2001, Abreu \& Vieira 2004). No nordeste, existem apenas o de Lopes (2002), em remanescente de Mata Atlântica semi-decidual, e os de Machado \& Lopes (2003, 2004) e Santos (2005), em áreas de caatinga. Para os ecossistemas desta região, até mesmo estudos de caso com espécies ornitófilas são poucos (Machado \& Sazima 1995, Locatelli \& Machado 1999, Machado \& Lopes 2000, Quirino \& Machado 2001, Siqueira Filho \& Machado 2001, 2004, Lopes et al. 2002).

A caatinga é um tipo vegetacional exclusivamente brasileiro (Aguiar et al. 2002), consistindo na maior zona fitogeográfica de Pernambuco (Andrade-Lima 1960), estendendo-se por cerca de $83 \%$ do estado (Hueck 1972) e por aproximadamente $800.000 \mathrm{~km}^{2}$ do território brasileiro (Ab’Sáber 1974, Aguiar et al. 2002). Apesar de ser o quarto maior tipo vegetacional do Brasil (Aguiar et al. 2002), as unidades de conservação neste ecossistema são poucas e pequenas (Araújo et al. 1995), sendo referidas apenas 22 (2,9\% do total da área de caatinga), das quais apenas 14 estão estritamente protegidas (Aguiar et al. 2002). A Reserva Particular do Patrimônio Natural Cantidiano Valgueiro está inserida no polígono das 27 áreas de extrema importância biológica da caatinga (Ministério do Meio Ambiente 2002), o qual demanda estudos urgentes voltados à conservação.

Tendo em vista a manutenção de processos ecológicos como a polinização, fundamentais para a conservação dos ecossistemas naturais, o presente trabalho teve como objetivo caracterizar os atributos florais e a fenologia de floração de espécies ornitófilas ocorrentes na referida reserva, bem como o comportamento e a fenologia das espécies de beija-flores visitantes, além das interações entre estes e as plantas.

\section{Material e métodos}

Área de estudo - O estudo foi realizado em uma área protegida de caatinga na Reserva Particular do Patrimônio Natural (RPPN) Cantidiano Valgueiro, Município de Floresta, Pernambuco (8³6'00”'S e 38 34'5” W), entre julho de 2002 e junho de 2003. A área da RPPN possui cerca de 298 ha e caracteriza-se por apresentar vegetação xerófila, decídua, com porte arbustivo-arbóreo, típica do alto sertão nordestino (Andrade-Lima 1960, 1981).

O clima na área é do tipo semi-árido (Bs s’h’ de Köppen) com estação seca entre os meses de abril e outubro e estação chuvosa entre novembro e março (Araújo et al. 1995 e Leal et al. 2003 para detalhes). A temperatura média anual é de, aproximadamente, $27^{\circ} \mathrm{C}$ (Sudene 1990) e a pluviosidade média anual de cerca de $520 \mathrm{~mm}$ (IPA, Posto de Floresta-PE, dados não publicados). Nos anos em que o estudo foi realizado a precipitação foi de 559,1, em 2002, e 447,8 mm, em 2003 (IPA, Posto de Floresta-PE).

Florística e fenologia de floração - As observações de campo foram realizadas ao longo de trilhas pré-existentes, sendo percorridos, a cada excursão, cerca de $5 \mathrm{~km}$. As excursões foram quinzenais e com duração de três a cinco dias cada, totalizando 665 horas de observações de campo. A cada excursão espécies vegetais com atributos florais ornitófilos (sensu Faegri \& Pijl 1979) foram registradas, marcadas e coletadas para posterior identificação. A cada visita foi feito o acompanhamento da fenologia de floração destas espécies, com exceção de Helicteres cf. velutina, representada na área percorrida por apenas um indivíduo, já em final de floração em outubro de 2002. Foi registrada a presença ou ausência de botões florais e flores para identificar o período de floração de cada uma, bem como os padrões fenológicos em níveis de espécie e de comunidade (Newstrom et al. 1994). As espécies que não apresentavam características florais ornitófilas, mas que receberam visitas de beija-flores, foram apenas registradas e identificadas.

Material botânico contendo ramos vegetativos e reprodutivos de cada espécie vegetal estudada foi coletado para identificação e depositado no Herbário do Departamento de Botânica da Universidade Federal de Pernambuco (UFP). Morfologia e biologia floral - Para cada espécie ornitófila foram registrados o tamanho, a cor e o tipo floral, o horário e a duração da antese, além do volume e da concentração de açúcares no néctar.

Para caracterizar a morfologia floral foram utilizadas de 4 a 15 flores frescas de cada espécie e medidos o comprimento interno do tubo da corola (Wolf et al. 1976, Buzato et al. 2000), excluindo lobos livres quando recurvados, e o diâmetro da abertura da corola (Lopes 2002).

O volume e a concentração de açúcares do néctar foram medidos em até cinco flores de cada espécie, de um a cinco 
indivíduos diferentes, ensacadas em pré-antese. As medidas foram realizadas com auxílio de micro-seringas (Microliter ${ }^{\circledR}$ $10 \mu \mathrm{L}$ ) e refratômetro de bolso (Atago® 0\%-50\%), no período da manhã, em torno de 3 a 4 horas após o início da antese (Lopes 2002).

Para verificar a existência de relação entre o comprimento da corola e o volume de néctar (sensu Arizmendi \& Ornelas 1990), bem como entre o comprimento da corola e a concentração de açúcares, foi realizada correlação de Spearman com auxílio do programa BioEstat 2.0 (Ayres et al. 2000).

Visitantes florais - Os visitantes florais foram estudados através de observações diretas ou com auxílio de binóculo, em diferentes horários do dia, ao longo das trilhas e/ou através de sessões de observações sobre grupos de indivíduos de cada espécie em floração. A cada visita de um beija-flor às flores das espécies ornitófilas, foram registrados o horário, a duração e o resultado da visita (polinização, inferida pelo contato com as partes reprodutivas da flor, ou pilhagem, quando o recurso era coletado sem que o animal contatasse as anteras e o estigma). Além disso, foram também verificados o modo de forrageamento (territorialista ou em rotas de captura/"traplining”) e a ocorrência de interações intra e interespecíficas (Feinsinger \& Colwell 1978, Arizmendi \& Ornelas 1990), sendo ainda anotados o número de avistamentos de cada espécie de beija-flor quando em visita às flores de uma determinada espécie.

Os beija-flores foram identificados no campo e as espécies confirmadas com auxílio de fotografias, de literatura especializada, como Grantsau (1989) e Sick (1997), além de consultas à coleção de aves do Departamento de Zoologia da Universidade Federal de Pernambuco. Medidas do comprimento do bico das espécies de beija-flores registradas foram retiradas de Grantsau (1989).

\section{Resultados}

Florística - Oito espécies ornitófilas, pertencentes a sete gêneros e cinco famílias, foram registradas na RPPN Cantidiano Valgueiro. Cactaceae foi predominante (37,5\%), com três espécies, juntamente com Bromeliaceae, representada por duas espécies (25\%) (tabela 1). Acanthaceae, Loranthaceae e Sterculiaceae foram representadas por uma espécie cada (tabela 1). Quatro espécies apresentaram hábito herbáceo (50\%), três eram arbustos (37,5\%) e uma hemiparasita (12,5\%) (tabela 1).

Fenologia de floração - Com exceção de Bromelia laciniosa e Neoglaziovia variegata, as demais espécies apresentaram pico de floração no período seco, de abril a outubro (figura 1). Os meses de fevereiro, março e abril foram os que apresentaram menor número de espécies florescendo simultaneamente (duas, duas e uma, respectivamente), ao passo que de maio a novembro floresceram três a cinco espécies concomitantemente (figura 1). Psittacanthus bicalyculatus, B. laciniosa e $N$. variegata floresceram por três meses, Ruellia asperula e Opuntia spp. floresceram por mais de quatro meses e Melocactus zehntneri apresentou flores o ano todo.

Morfologia e biologia floral - A maioria das espécies apresentou flores vermelhas (cerca de $75 \%$ ), sendo o tipo tubo encontrado em todas as espécies ornitófilas registradas (tabela 1 ). O comprimento médio da corola foi de 20,2 $\pm 5,6 \mathrm{~mm}$, variando de $11,2 \pm 1,2 \mathrm{~mm}$ (B. laciniosa) a 27,24 $\pm 0,48 \mathrm{~mm}$ (Helicteres cf. velutina) (tabela 1 ), enquanto o diâmetro médio da abertura da corola foi de 5,8 $\pm 4,42 \mathrm{~mm}$, variando de $2,5 \pm 0,6 \mathrm{~mm}$ (P. bicalyculatus) a $16 \pm 8,4 \mathrm{~mm}$ (Opuntia palmadora) (tabela 1).

Na maioria das espécies a antese teve início ao amanhecer, exceto em $P$. bicalyculatus, $M$. zehntneri e R. asperula, cuja abertura floral iniciou às $2 \mathrm{~h} 30$ - 3h00, $14 \mathrm{~h} 30$ e 15h00, respectivamente. Bromelia laciniosa, Neoglaziovia variegata e Opuntia inamoena apresentaram flores com duração de cerca de 12 horas, enquanto em outras quatro espécies as flores duraram mais de um dia: Helicteres cf. velutina, Opuntia palmadora, Ruellia asperula (2 dias) e Psittacanthus bicalyculatus (3 dias). As flores de Melocactus zehntneri duraram apenas quatro horas (14h30 - 18h30). A quantidade de flores abertas por indivíduo, por dia, variou de uma até dezenas, como em $H$. cf. velutina (tabela 1).

O volume de néctar variou de $22 \mu \mathrm{L}$ (P. bicalyculatus) a $41 \pm 1,18 \mu \mathrm{L}$ (M. zehntneri), enquanto a concentração de açúcares variou de $18 \%$ (P. bicalyculatus) a 33,5\% (R. asperula) (tabela 1). Não houve correlação entre o comprimento da corola e o volume de néctar ( $r=-0,3143, P=0,5441 ; N=6$ spp.), nem entre o comprimento e a concentração de açúcares ( $r=0,4643, P=0,2939 ; N=7$ spp.) das flores das espécies estudadas.

Visitantes florais - Foram registradas cinco espécies de beija-flores na comunidade estudada: Phaethornis gounellei Boucard, 1891 (Phaethornithinae), Chlorostilbon aureoventris (d'Orbigny \& Lafresnaye, 1838), Chrysolampis mosquitus (Linnaeus, 1758), Eupetomena macroura (Gmelin, 1788) e Heliomaster squamosus (Temminck, 1823) (Trochilinae). Dentre estas espécies, C. aureoventris foi a mais abundante e freqüente, tendo sido registrada na comunidade ao longo de todo o ano e visitado flores de todas as espécies ornitófilas estudadas (figura 1, tabela 2). As demais 


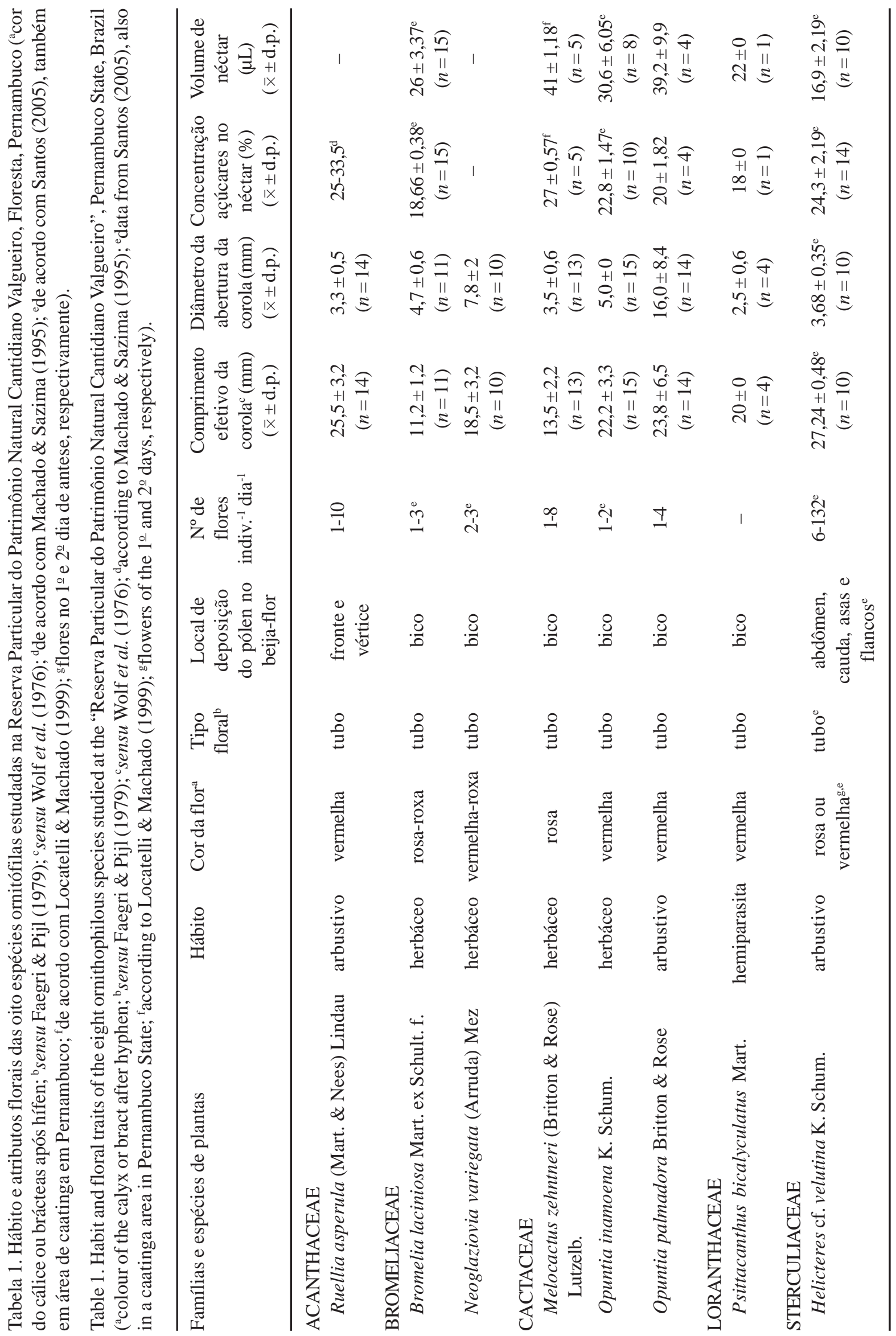


espécies foram observadas em períodos restritos do ano (figura 1) e visitaram flores de poucas espécies (tabela 2), como foi o caso de E. macroura (janeiro e maio a julho) e H. squamosus (fevereiro e maio a julho), registradas em ambas as estações, e $C$. mosquitus (fevereiro e março) e $P$. gounellei (abril, agosto e outubro), avistadas em apenas uma das estações, chuvosa e seca, respectivamente (figura 1).

A maioria das espécies de plantas teve suas flores visitadas e polinizadas por mais de uma espécie de

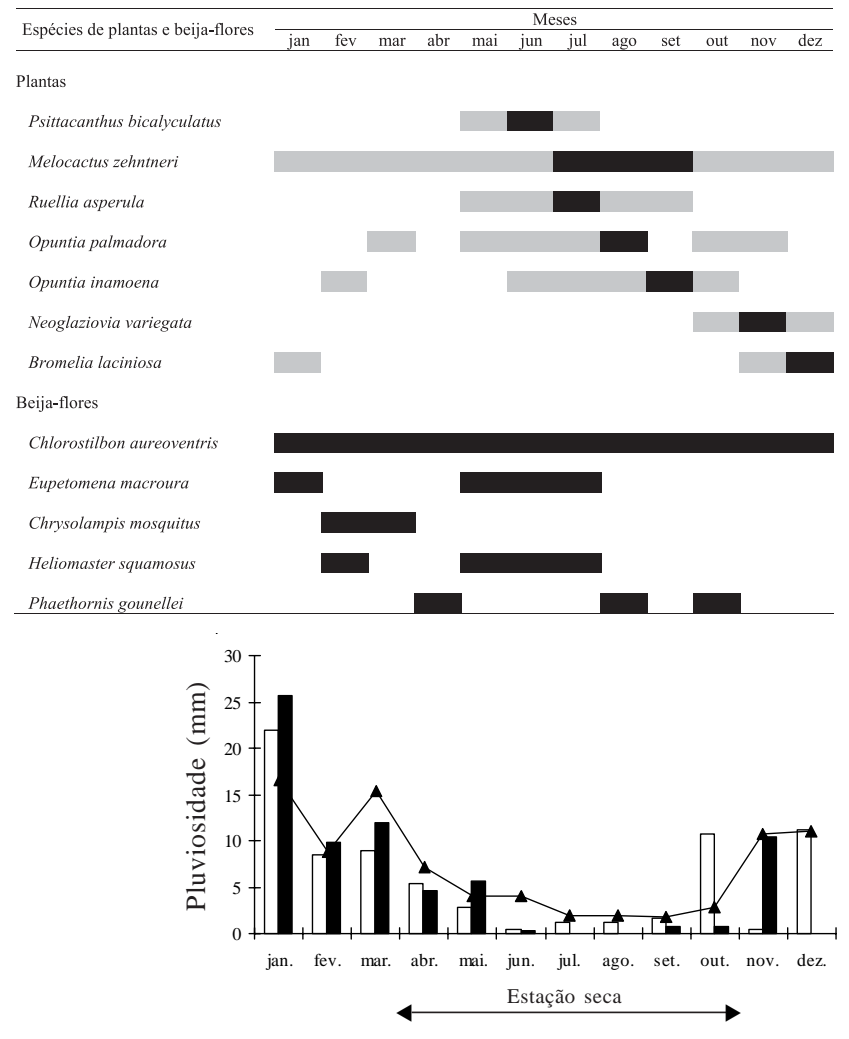

Figura 1. Fenologia de floração de sete espécies ornitófilas, meses em que foram registradas cada uma das cinco espécies de beija-flores e pluviosidade média mensal na Reserva Particular do Patrimônio Natural Cantidiano Valgueiro, Pernambuco, no período de julho de 2002 a junho de 2003 (fonte de dados pluviométricos básicos: IPA, Posto Floresta$\mathrm{PE}$ ). Picos de floração (mais de 50\% dos indivíduos em floração) em preto; $\square$ = pluviosidade media mensal em 2002; $\boldsymbol{\square}=$ em 2003; $\boldsymbol{\Delta}$ = em 12 anos.

Figure 1. Flowering phenology of seven ornithophilous species, monthly registration of the five hummingbird species, and monthly average rainfall at the "Reserva Particular do Patrimônio Natural Cantidiano Valgueiro”, Pernambuco State, Brazil, from July 2002 to June 2003 (rainfall data from IPA, Posto Floresta-PE). Flowering peaks (more than $50 \%$ of the individuals in flower) in black; $\square=$ monthly average rainfall in 2002; $\mathbf{\square}=$ in 2003; $\boldsymbol{\Delta}=$ in 12 years. beija-flor (tabela 2). Bromelia laciniosa foi visitada por quatro das espécies de beija-flores e Melocactus zehntneri por duas, sendo Chlorostilbon aureoventris considerado como polinizador freqüente e efetivo de ambas. Ruellia asperula e Neoglaziovia variegata receberam visitas apenas de $C$. aureoventris (tabela 2).

Em todas as visitas registradas, os beija-flores atuaram como polinizadores, contatando anteras e estigmas, exceto em botões florais de Psittacanthus bicalyculatus, nos quais $C$. aureoventris e Heliomaster squamosus atuaram como pilhadores. Espécies como M. zehntneri e P. bicalyculatus foram também visitadas legitimamente por borboletas. No entanto, estas nem sempre contataram as estruturas reprodutivas, além das visitas terem sido menos freqüentes que as dos beija-flores.

Chlorostilbon aureoventris, $H$. squamosus e Chrysolampis mosquitus visitaram também flores que não apresentavam características descritas para a síndrome de ornitofilia como Caesalpinia pyramidalis Tul. (Caesalpinaceae), Encholirium spectabile Mart. (Bromeliaceae), Ipomoea sp. (Convolvulaceae), Cnidoscolus cf. urens (L.) Arthur (Euphorbiaceae), Melochia tomentosa L. (Sterculiaceae) e Oxalis sp. (Oxalidaceae), todas em floração na estação chuvosa, principalmente entre fevereiro e abril. Ao visitarem flores destas espécies, comportaram-se como pilhadores nas três primeiras, retirando néctar sem contatar as estruturas reprodutivas das flores, e como polinizadores nas três outras.

Os Trochilinae apresentaram comportamentos desde generalista até territorialista ou ainda forragearam em rotas de captura ("traplining”) (tabelas 2-3). Chlorostilbon aureoventris atuou como territorialista ou "trapliner" dependendo da espécie visitada (sensu Feinsinger \& Colwell 1978) (tabelas 2-3). Chrysolampis mosquitus visitou flores de Bromelia laciniosa e Opuntia inamoena com baixa freqüência de visitas, tendo sido considerado, em ambas, polinizador ocasional (tabela 2). Phaethornis gounellei, apesar de registrado na área de estudo, não foi observado visitando flores de nenhuma espécie estudada. Eupetomena macroura visitou quatro espécies, sempre com menor frequência que $C$. aureoventris (tabela 2) e atuou como polinizador ocasional em todas elas. Heliomaster squamosus também visitou flores de quatro espécies, sendo considerado polinizador efetivo em apenas uma (Psittacanthus bicalyculatus), por contatar partes florais reprodutivas, dividindo também recursos dessas quatro espécies com C. aureoventris (tabela 2). 
Chlorostilbon aureoventris e H. squamosus sobrepuseram-se no uso de néctar de quatro espécies, atuando inclusive como polinizadores freqüentes e efetivos de $P$. bicalyculatus (tabela 2).

O bico dos beija-flores foi o local mais freqüente de deposição de pólen, tendo sido observado contato de

Tabela 2. Número de visitas e comportamento das espécies de beija-flores às flores de sete espécies ornitófilas na Reserva Particular do Patrimônio Natural Cantidiano Valgueiro, Floresta, Pernambuco ( ${ }^{1}$ plantas e beija-flores listados em ordem alfabética; abreviaturas das espécies de beija-flores: Chl. aur. =Chlorostilbon aureoventris; Chr. mos. = Chrysolampis mosquitus; Eu. mac. $=$ Eupetomena macroura; He. squ. $=$ Heliomaster squamosus; ${ }^{2}$ tempo em que as visitas foram monitoradas e contabilizadas; $\mathrm{TP}=$ “Traplining”; TR = Territorial; OC = visitas ocasionais).

Table 2. Number of visits and behaviour of the hummingbirds when visiting flowers of seven ornithophilous species at the "Reserva Particular do Patrimônio Natural Cantidiano Valgueiro", Pernambuco State, Brazil ('plants and hummingbirds in alphabetical order; abbreviation of the hummingbird spp.: Chl. aur. = Chlorostilbon aureoventris; Chr. mos. $=$ Chrysolampis mosquitus; Eu. mac. = Eupetomena macroura; He. squ. $=$ Heliomaster squamosus; ${ }^{2}$ total time of monitoring and counting of the visits; TP = "Traplining"; TR =Territorial; OC = occasional visits).

\begin{tabular}{|c|c|c|c|c|c|}
\hline \multirow[t]{2}{*}{ Espécies de plantas ${ }^{1}$} & \multicolumn{4}{|c|}{ Beija-flores $^{1}$} & \multirow{2}{*}{$\begin{array}{c}\text { Tempo de observ. } \\
\text { (h) }\end{array}$} \\
\hline & Chl. aur. & Chr. mos. & Eu. mac & Не. squ. & \\
\hline Bromelia laciniosa & $56(\mathrm{TP})$ & $1(\mathrm{OC})$ & $4(\mathrm{OC})$ & $1(\mathrm{OC})$ & 14,5 \\
\hline Melocactus zehntneri & $92(\mathrm{TR})$ & & $7(\mathrm{OC})$ & & 20 \\
\hline Neoglaziovia variegata & $3(\mathrm{TP})$ & & & & 8 \\
\hline Opuntia inamoena & $8(\mathrm{TP})$ & $1(\mathrm{OC})$ & & $2(\mathrm{OC})$ & 15 \\
\hline Opuntia palmadora & $58(\mathrm{TP})$ & & $4(\mathrm{OC})$ & $4(\mathrm{OC})$ & 19,5 \\
\hline Psittacanthus bicalyculatus & 70 (ТP) & & $5(\mathrm{OC})$ & 19 (ТР) & 26,5 \\
\hline Ruellia asperula & $18(\mathrm{TP})$ & & & & 12 \\
\hline $\begin{array}{l}\mathrm{N}^{\circ} \text { ssp. em que atuou como polinizador } \\
\text { freqüente e efetivo }\end{array}$ & 7 & 0 & 0 & 1 & \\
\hline $\begin{array}{l}\mathrm{N}^{\circ} \text { spp. em que houve sobreposição no } \\
\text { uso de recursos com demais visitantes efetivos }\end{array}$ & 1 & 0 & 0 & 1 & \\
\hline $\begin{array}{l}\text { Total de horas em que as visitas foram } \\
\text { contabilizadas }\end{array}$ & & & & & 115,5 \\
\hline
\end{tabular}

Tabela 3. Espécies de beija-flores observadas na Reserva Particular do Patrimônio Natural Cantidiano Valgueiro, Floresta, Pernambuco, com respectivos comprimentos dos bicos, fidelidade e papel desempenhado na comunidade estudada. [*papel não inferido, visto somente em três ocasiões na comunidade; ${ }^{1}$ comprimento do cúlmen exposto a partir de Grantsau (1989); ${ }^{2}$ sensu Feinsinger \& Colwell (1978)].

Table 3. Hummingbirds observed at the "Reserva Particular do Patrimônio Natural Cantidiano Valgueiro", Pernambuco State, Brazil, with their bill length, fidelity and role in the studied community [*function not inferred, species observed only three times at the community; ${ }^{1}$ lenght of the exposed culmen from Grantsau (1989); ${ }^{2}$ sensu Feinsinger \& Colwell (1978)].

\begin{tabular}{lccc}
\hline Beija-flores & $\begin{array}{c}\text { Comprimento } \\
\text { do bico } \\
(\mathrm{mm})\end{array}$ & $\begin{array}{c}\text { Fidelidade } \\
\text { à comunidade } \\
\text { estudada }^{2}\end{array}$ & Papel na comunidade estudada ${ }^{2}$ \\
\hline $\begin{array}{l}\text { PHAETHORNITHINAE } \\
\text { Phaethornis gounellei Boucard, 1891 }\end{array}$ & 28,0 & Secundário & $*$ \\
$\begin{array}{l}\text { TROCHILINAE } \\
\quad \text { Chlorostilbon aureoventris (d'Orbigny \& } \\
\quad \text { Lafresnaye, 1838) }\end{array}$ & $19,3-20,0$ & Principal & Rondas de alto ganho e Territorialista \\
$\quad \begin{array}{l}\text { Chrysolampis mosquitus (Linnaeus, 1758) } \\
\text { Eupetomena macroura (Gmelin, 1788) }\end{array}$ & $\begin{array}{c}\text { 12-14,5 } \\
\text { Heliomaster squamosus (Temminck, 1823) }\end{array}$ & $\begin{array}{c}\text { Secundário } \\
\text { Secundário } \\
\text { Secundário }\end{array}$ & $\begin{array}{c}\text { Generalista } \\
\text { Generalista }\end{array}$ \\
\end{tabular}


pólen com esta região do corpo dos beija-flores em seis (75\%) das oito espécies de plantas estudadas (tabela 1). Exceto para Phaethornis gounellei e Heliomaster squamosus, o bico das demais espécies de beija-flores pode ser considerado curto ( $<25 \mathrm{~mm}$ sensu Feinsinger et al. 1987; tabela 3).

\section{Discussão}

Composição florística e hábito - O número de espécies ornitófilas registrado durante o período de estudo na RPPN Cantidiano Valgueiro (8 spp.) foi menor que aquele encontrado em remanescentes de Mata Atlântica (Sazima et al. 1995, 1996, Buzato et al. 2000, Lopes 2002) e em outras áreas neotropicais (Snow \& Snow 1972, 1980, Feinsinger et al. 1987, Linhart et al. 1987, Dziedzioch et al. 2003), onde este número variou de 22 a 72 espécies. De modo geral, a diversidade de espécies vegetais em florestas secas é, de fato, menor quando comparada a florestas úmidas (Gentry 1995). Por sua vez, o número de espécies ornitófilas da RPPN foi similar ao registrado em áreas com vegetação semidecídua a decídua (Kodric-Brown et al. 1984, Arizmendi \& Ornelas 1990, Machado \& Lopes 2003, Araújo \& Sazima 2003), onde este variou de 6 a 13 espécies. Porém, o número aqui encontrado foi cerca da metade do registrado em áreas de caatinga em Buíque, Pernambuco, onde foram observadas 12 e 18 espécies ornitófilas (Santos 2005). Estas áreas estudadas por Santos (2005), entretanto, apresentam fisionomias distintas da área do presente estudo, com muitos elementos de campos rupestres (ver Rodal et al. 1998).

No que diz respeito à distribuição de espécies ornitófilas em variados gêneros e famílias como encontrados no presente estudo, este padrão tem sido também registrado para outros ecossistemas (Snow \& Snow 1972, 1980, 1986, Arizmendi \& Ornelas 1990, Sazima et al. 1996, Buzato et al. 2000, Lopes 2002). O fato de Cactaceae ter sido a família com maior número de espécies ornitófilas $(37,5 \%)$ pode decorrer da associação de fatores como sua grande representatividade em florestas secas (Gentry 1995, Griz \& Machado 2001, Machado \& Lopes 2003, 2004) e da ornitofilia ser bastante comum no grupo (Machado \& Lopes 2004). Bromeliaceae, a segunda família mais representativa em número de espécies ornitófilas no presente estudo (25\%), é citada por diversos autores como sendo responsável pelo maior percentual de espécies ornitófilas em diferentes comunidades vegetacionais como a mata atlântica (Snow \& Snow
1986, Sazima et al. 1995, 1996, Buzato et al. 2000, Lopes 2002), florestas serranas (ou brejos de altitude) (Siqueira Filho \& Machado 2004), florestas andinas (Dziedzioch et al. 2003) e caatinga (Santos 2005).

A predominância do hábito herbáceo na maior parte das espécies estudadas também foi registrada em outras comunidades incluindo formações florestais, tanto úmidas (Toledo 1975), como semideciduais (Araújo \& Sazima 2003, Abreu \& Vieira 2004), campos rupestres (Vasconcelos \& Lombardi 2001) e caatinga (Santos 2005). Porém, epífitas e lianas foram mais freqüentes entre as espécies ornitófilas em vários outros estudos enfocando comunidades florestais tanto úmidas (Snow \& Snow 1986, Sazima et al. 1996, Buzato et al. 2000, Dziedzioch et al. 2003), quanto semideciduais (Arizmendi \& Ornelas 1990, Lopes 2002). Uma vez que o conjunto de estudos referidos acima inclui florestas úmidas e semideciduais, além de campos rupestres e caatinga, parece não haver associação entre hábito predominante em espécies ornitófilas e tipo vegetacional. Fenologia de floração - Apesar do número reduzido de espécies, a comunidade ornitófila apresentou floração contínua (sensu Newstrom et al. 1994), sendo isso fundamental para a manutenção das espécies de beijaflores (Feinsinger 1978, Buzato et al. 2000, Lopes 2002). Apesar de em todos os meses do ano terem sido registradas espécies ornitófilas florescendo, houve diferença entre as estações. A maioria das espécies floresceu e teve pico de floração na estação seca. Vários estudos referem-se à sazonalidade na floração de plantas ornitófilas (Wolf 1970, Wolf et al. 1976, Koptur et al. 1988, Buzato et al. 2000), principalmente em florestas secas (Koptur et al. 1988, Lopes 2002), apontando o período chuvoso como o que apresenta maior número de espécies floridas (Sazima et al. 1996, Buzato et al. 2000, Lopes 2002). Entretanto, outros trabalhos registram a estação seca como a que apresenta maior número de espécies ornitófilas floridas (Wolf 1970, Stiles 1978, Arizmendi \& Ornelas 1990, Santos 2005), concordando com o presente estudo.

Com exceção de Melocactus zehntneri, que floresceu durante todo o ano, apresentando padrão de floração contínuo, as demais seis espécies apresentaram padrão anual (sensu Newstrom et al. 1994), o que, de fato, era esperado para uma comunidade de plantas ornitófilas (Stiles 1978). Mesmo o estudo tendo sido realizado em apenas um ano, o padrão anual observado para essas seis espécies pode ser confirmado, uma vez que todas elas foram observadas florescendo anualmente tanto na área de estudo, quanto em outras áreas de 
caatinga (A. Lopes \& I. Machado, dados não publicados) de maneira que a possilibilidade de alguma ter padrão supra-anual pode ser excluída.

Morfologia e biologia floral - O predomínio de espécies com flores vermelhas e tubulosas (encontrado em todas as espécies) é característico de comunidades de plantas ornitófilas (Sazima et al. 1995, 1996, Buzato et al. 2000, Lopes 2002). A maioria das espécies ornitófilas no local de estudo (cerca de 75\%) apresentou flores com corola curta (comprimento do tubo $\leq 25 \mathrm{~mm}$ ) e foi visitada, principalmente, por beija-flores de bico curto (culmen externo < 25 mm sensu Feinsinger et al. 1987), situação semelhante à observada por Linhart et al. (1987), Feinsinger et al. (1987) e Santos (2005). Dentre as cinco espécies de beija-flores registradas na comunidade estudada, Heliomaster squamosus e Phaethornis gounellei apresentam bicos longos ( $\geq 28 \mathrm{~mm}$ sensu Feinsinger et al. 1987) e as demais, bicos curtos ( $<25 \mathrm{~mm}$ ), de maneira que o predomínio de espécies com corolas curtas permite acesso de néctar a todas as espécies.

A concentração média de açúcares no néctar das espécies ornitófilas está de acordo com o encontrado por Baker (1975), Sazima et al. (1995, 1996), Buzato et al. (2000) e Lopes (2002) para "flores de beijaflores”. De maneira semelhante ao registrado por Buzato (1995) e Santos (2005), o volume de néctar não foi correlacionado com o comprimento da corola, diferindo, entretanto, dos registros de Arizmendi \& Ornelas (1990) e Lopes (2002), os quais verificaram correlação positiva entre estes dois atributos.

$\mathrm{Na}$ comunidade estudada tanto em flores com diâmetro de abertura pequeno quanto em flores com diâmetros maiores a deposição de pólen ocorreu, principalmente, no bico. Resultado semelhante também foi observado por Sazima et al. (1995, 1996), Buzato et al. (2000) e Lopes (2002), estando diretamente relacionado a flores tubulosas (Buzato et al. 2000). Neste sentido, mesmo em Opuntia palmadora, cujo diâmetro da abertura da corola foi o maior da comunidade, a deposição de pólen ocorreu no bico dos beija-flores visitantes, resultado encontrado também por Locatelli \& Machado (1999). Nas únicas espécies em que a deposição de pólen no corpo dos beija-flores não ocorreu no bico, mas sim no vértice e fronte (Ruellia asperula) ou no abdômen, cauda, asas e flancos (Helicteres cf. velutina), os diâmetros da abertura das corolas estiveram entre os menores da comunidade e os comprimentos das flores entre os maiores. Nestes casos, a deposição de pólen pode ter sido influenciada tanto pelo comprimento do tubo quanto pela maior exposição das estruturas reprodutivas. Diante disso, o comprimento do tubo da corola, bem como das estruturas reprodutivas, foram, no conjunto de espécies aqui estudadas, mais determinantes do local de deposição de pólen no corpo dos beija-flores do que o diâmetro da abertura da corola. Entretanto, o uso de um ou poucos atributos morfológicos florais não explica as complexas relações entre a procura de flores por beija-flores (Temeles et al. 2002).

Quanto ao número de flores por indivíduo e a quantidade de néctar produzido por flor, observou-se, na comunidade estudada, dois dos quatro tipos florais propostos por Feinsinger \& Colwell (1978) e Buzato et al. (2000): flores "esparsas e ricas em néctar" (<30 flores indivíduo ${ }^{-1} \mathrm{dia}^{-1}, \geq 20 \mu \mathrm{L}$ néctar flor ${ }^{-1}$ e "agrupadas e moderadamente ricas em néctar" ( $\geq 30$ flores indivíduo $^{-1}$ dia $^{-1},<20 \mu \mathrm{L}$ néctar flor $\left.{ }^{-1}\right)$. Este último representado apenas por $H$. cf. velutina que, segundo dados de Santos (2005), se enquadraria neste tipo. Este fato assemelha-se ao encontrado por Buzato et al. (2000), em floresta úmida, e Santos (2005), em área de caatinga, os quais verificaram, respectivamente, que cerca de $60 \%$ e $67 \%$ das espécies ornitófilas estudadas enquadravam-se no tipo "esparsas e ricas". Este padrão favorece o comportamento de forrageamento do tipo "traplining" com rondas de alto ganho, comportamento este observado em Chlorostilbon aureoventris (tabela 3) em várias espécies ornitófilas.

Visitantes florais - Apenas Chlorostilbon aureoventris foi observado ao longo de todo o ano na comunidade estudada, sendo considerado como polinizador "principal”, sendo as demais espécies "secundárias" (sensu Feinsinger \& Colwell 1978) por ocorrerem apenas em alguns meses do ano (tabela 3). Esta espécie foi também considerada como polinizador efetivo das sete espécies de plantas estudadas, uma vez que contatou as estruturas reprodutivas das mesmas. Em Melocactus zehntneri e Opuntia palmadora, C. aureoventris não foi o único beija-flor a polinizálas, mas foi o mais freqüente, tendo sido, entretanto, o único polinizador dessas espécies em outra área de caatinga em Pernambuco (Locatelli \& Machado 1999). Já Ruellia asperula, que no presente estudo foi visitada apenas por C. aureoventris, foi, segundo Machado \& Sazima (1995), vistada por outras cinco espécies de beija-flores em outra área de caatinga, também em Pernambuco. Apesar do elevado número de espécies visitando as flores de $R$. asperula, as autoras referem-se apenas a Eupetomena macroura como polinizador efetivo, as demais visitando principalmente de modo ilegítimo. 
Chlorostilbon aureoventris (Trochilinae) devido ao seu comportamento de visita, freqüente, pois foi registrado durante o ano todo na comunidade, e efetivo, pois polinizou todas as espécies ornitófilas, foi considerado como espécie "organizadora" da guilda (sensu Feinsinger \& Colwell 1978). Entretanto, em outros estudos, espécies de Phaethornithinae são apontadas como organizadoras das comunidades (Sazima et al. 1995, Buzato et al. 2000, Lopes 2002). Apesar de não ser comum um Trochilinae assumir este papel, Feinsinger (1976) e Araújo \& Sazima (2003) também registraram espécies de Trochilinae [Amazilia saucerottei (DeLattre \& Bourcier, 1846) e Hylocharis chrysura (Shaw, 1812), respectivamente] organizando assembléias de plantas ornitófilas. É possível que, de fato, a comunidade aqui estudada não suportasse manter um Phaethornithinae como espécie organizadora, devido à escassez ou ausência de plantas com os requisitos energéticos e as características florais associadas aos mesmos, como grande número de espécies ornitófilas e flores com corolas longas.

Phaethornis gounellei, espécie endêmica do Nordeste (Nascimento 2000), não foi observada em flores, tendo sido apenas registrada sua presença na área de estudo nos meses de abril, agosto e outubro, não sendo possível, portanto, enquadrá-la na classificação proposta por Feinsinger \& Colwell (1978) (tabela 3). De acordo com Silva et al. (2003), $P$. gounellei ocorre, preferencialmente, em "ambientes florestais” no domínio da caatinga e com pouca perturbação antrópica, situação não encontrada na área estudada, a qual tem porte médio a baixo e foi transformada apenas recentemente em uma reserva, tendo indícios de perturbação antrópica.

As visitas realizadas na estação chuvosa por algumas espécies de beija-flores a flores de espécies não ornitófilas, período este em que a maioria das espécies ornitófilas não estava em floração, corroboram os estudos de Machado \& Lopes (2003, 2004) mencionando que espécies não ornitófilas constituem importante recurso para a permanência e diversidade das espécies de beija-flores nas comunidades de caatinga.

A comunidade de espécies ornitófilas da RPPN Cantidiano Valgueiro apresenta, portanto, alguns aspectos que diferem da maioria dos estudos com o mesmo enfoque, realizados em outros ecossistemas, como o baixo número de espécies tipicamente ornitófilas que compõem a comunidade e a organização da comunidade ornitófila por uma espécie de Trochilinae (Chlorostilbon aureoventris). Estes fatos refletem, provavelmente, a forte influência das condições climáticas, tanto na diversidade, quanto na disponibilidade de recursos durante o ano. No entanto, estudos em outras áreas de caatinga e com maior tempo de observação são imprescindíveis para que se possa avaliar se os dados aqui registrados constituem, ou não, um padrão para a caatinga.

Agradecimentos - À MSc. Mary Janice Santos, pela ajuda em campo, sugestões e valiosas comunicações pessoais sobre dados ainda não publicados. Aos dois assessores anônimos, pelas sugestões relevantes ao trabalho. À Biol. Marlene Barbosa, Curadora do Herbário UFP, pelo processamento do material botânico junto ao Herbário UFP; às Sras. Elza Carvalho e Adália Valgueiro, que permitiram que este trabalho fosse desenvolvido na RPPN-Cantidiano Valgueiro; ao Dr. Severino Mendes Júnior, pela gentileza quando das consultas à coleção de aves do Departamento de Zoologia da UFPE. A Tarcila Nadia, Virgínia Leite e André Santos pela ajuda em várias etapas na elaboração do trabalho. À Capes e ao CNPq pelo apoio financeiro a F. Leal (Bolsa de mestrado) e a A. Lopes e I. Machado (Bolsas de Produtividade em Pesquisa), respectivamente.

\section{Referências bibliográficas}

ABREU, C.R.M \& VIEIRA, M.F. 2004. Os beija-flores e seus recursos florais em um fragmento florestal de Viçosa, sudeste brasileiro. Instituto de Ciências Biológicas, Universidade Federal de Minas Gerais, Minas Gerais. Lundiana 5:129-134.

AB’SÁBER, A.N. 1974. O domínio morfoclimático semi-árido das caatingas brasileiras. Instituto de Geografia, Universidade de São Paulo, São Paulo.

AGUIAR, J., LACHER, T. \& SILVA, J.M.C. 2002. The caatinga. In Wilderness - Earth's Last Wild Places (P.R. Gil, ed.). Cemex, Cidade do México, p.174-181.

ANDRADE-LIMA, D. 1960. Estudos Fitogeográficos de Pernambuco. Arquivos do Instituto de Pesquisas Agronômicas de Pernambuco 5:305-341.

ANDRADE-LIMA, D. 1981. The caatingas dominium. Revista Brasileira de Botânica 4:149-153.

ARAÚJO, A.C. \& SAZIMA, M. 2003. The assemblage of flowers visited by hummingbirds in the "capões" of Southern Pantanal, Mato Grosso do Sul, Brazil. Flora 198:427-435.

ARAÚJO, E.L., SAMPAIO, E.V.S.B. \& RODAL, M.J.N. 1995. Composição florística e fitossociológica de três áreas de caatinga. Revista Brasileira de Biologia 55:595-607.

ARIZMENDI, M.C. \& ORNELAS, J.F. 1990. Hummingbirds and their floral resources in a tropical dry forest in Mexico. Biotropica 22:172-180. 
AYRES, M., AYRES JÚNIOR, M., AYRES, D.L. \& SANTOS, A.S. 2000. BioEstat 2.0: Aplicações estatísticas nas áreas das ciências biológicas e médicas. Sociedade Civil Mamirauá, CNPq, Belém.

BAKER, H.G. 1975. Sugar concentrations in nectar from hummingbird flowers. Biotropica 7:37-41.

BAWA, K.S. 1990. Plant-pollinator interactions in a tropical rain forest. Annual Review of Ecology and Systematics 21:339-422.

BROWN, J.H. \& BOWERS, M.A. 1985. Community organization in hummingbirds: relationships between morphology and ecology. The Auk 102:251-269.

BUZATO, S. 1995. Estudo comparativo de flores polinizadas por beija-flores em três comunidades da Mata Atlântica no Sudeste do Brasil. Tese de doutorado, Universidade Estadual de Campinas, Campinas.

BUZATO, S., SAZIMA, M. \& SAZIMA, I. 2000. Hummingbird-pollinated floras at three Atlantic Forest sites. Biotropica 32:824-841.

DZIEDZIOCH, C., STEVENS, A.D. \& GOTTSBERGER, G. 2003. The hummingbird plant community of a tropical montane rain forest in southern Ecuador. Plant Biology 5:331-337.

ENDRESS, P.K. 1994. Diversity and evolutionary biology of tropical flowers. Cambridge University Press, Cambridge.

FAEGRI, K. \& PIJL, L. VAN DER. 1979. The principles of pollination ecology. Pergamon Press, London.

FEINSINGER, P. 1976. Organization of a tropical guild of nectarivorous birds. Ecological Monographs 46:257-291.

FEINSINGER, P. 1978. Ecological interactions between plants and hummingbirds in a successional tropical community. Ecological Monographs 48:269-287.

FEINSINGER, P. 1983. Coevolution and pollination. In Coevolution (D.J. Futuyma \& M. Slatkin, eds.). Sinauer Associates, Sunderland, p.282-310.

FEINSINGER, P. \& COLWELL, R.K. 1978. Community organization among neotropical nectar-feeding birds. American Zoologist 18:779-795.

FEINSINGER, P., BEACH, J.H., LINHART, Y.B., BUSBY, W.H. \& MURRAY, K.G. 1987. Disturbance, pollinator predictability, and pollination success among Costa Rican cloud forest plants. Ecology 68:1294-1305.

FISCHER, E.A. 1996. Polinização por beija-flores In Anais do V Congresso Brasileiro de Ornitologia, Campinas, p.85-90.

GENTRY, A. 1995. Diversity and floristic composition of Neotropical dry forests. In Seasonally dry tropical forest (S. Bullock, H.A. Money \& E. Medina, eds.). Cambridge University Press, Cambridge, p.146-194.

GRANTSAU, R. 1989. Os beija-flores do Brasil. Expressão e Cultura, Rio de Janeiro.

GRIZ, L.M.S. \& MACHADO, I.C.S. 2001. Fruiting phenology and seed dispersal syndromes in caatinga, a tropical dry forest in the Northeast of Brazil. Journal of Tropical Ecology 17:303-321.
HUECK, K. 1972. As florestas da América do Sul: Ecologia composição e importância econômica. Polígono, São Paulo.

KODRIC-BROWN, A., BROWN, J., BYERS, GS. \& GORI, D.F. 1984. Organization of a tropical island community of hummingbirds and flowers. Ecology 65:1358-1368.

KOPTUR, S., HARBER, W.A., FRANKIE, G.W. \& BAKER, H.G. 1988. Phenological studies of shrub and treelet species in tropical cloud forests of Costa Rica. Journal of Tropical Ecology 4:323-346.

LASPRILLA, L.R. \& SAZIMA, M. 2004. Interacciones plantacolibrí en tres comunidades vegetales de la parte suroriental del Parque Nacional Natural Chiribiquete, Colômbia. Ornitologia Neotropical 15:183-190.

LEAL, I.R., TABARELLI, M. \& SILVA, J.M.C. (orgs.). 2003. Ecologia e conservação da caatinga. Editora Universitária, Universidade Federal de Pernambuco, Recife.

LINHART, Y.B., BUSBY, W.H., BEACH, J.H. \& FEINSINGER, P. 1987. Forager behavior, pollen dispersal, and inbreeding in two species of hummingbird-pollinated plants. Evolution 41:679-682.

LOCATELLI, E. \& MACHADO, I.C.S. 1999. Comparative study of the floral biology of two ornithophilous species of Cactaceae: Melocactus zehntneri and Opuntia palmadora. Bradleya 17:75-85.

LOPES, A.V.F. 2002. Polinização por beija-flores em remanescente da Mata Atlântica Pernambucana, Nordeste do Brasil. Tese de doutorado, Universidade Estadual de Campinas, Campinas.

LOPES, A.V., VOGEL, S. \& MACHADO, I.C. 2002. Secretory trichomes, a substitutive floral nectar source in Lundia A. DC. (Bignoniaceae), a genus lacking a functional disc. Annals of Botany 90:169-174.

MACHADO, I.C. \& LOPES, A.V. 2000. Souroubea guianensis Aubl.: quest for its legitimate pollinator and the first record of tapetal oil in the Marcgraviaceae. Annals of Botany 85:705-711.

MACHADO, I.C. \& LOPES, A.V. 2003. Recursos florais e sistemas de polinização e sexuais em caatinga. In Ecologia e conservação da caatinga (I.R. Leal, M. Tabarelli \& J.M.C. Silva, orgs.). Editora Universitária, Universidade Federal de Pernambuco, Recife, p.515-563.

MACHADO, I.C. \& LOPES, A.V. 2004. Floral traits and pollination systems in the caatinga, a Brazilian tropical dry forest. Annals of Botany 94:365-376.

MACHADO, I.C.S. \& SAZIMA, M. 1995. Biologia da polinização e pilhagem por beija-flores em Ruellia asperula Lindau (Acanthaceae) na caatinga, Nordeste Brasileiro. Revista Brasileira de Botânica 18:27-33.

MINISTÉRIO DO MEIO AMBIENTE 2002. Avaliação e ações prioritárias para a conservação da biodiversidade da caatinga. Secretaria de Biodiversidade e Floresta, Brasília.

NASCIMENTO, J.L.X. 2000. Estudo comparativo da avifauna em duas estações ecológicas da caatinga: Aiuaba e Seridó. Melopsittacus 3:12-35. 
NEWSTROM, L.E., FRANKIE, G.W. \& BAKER, H.G. 1994. A new classification for plant phenology based on flowering patterns in Lowland Tropical Rain Forest trees at La Selva, Costa Rica. Biotropica 26:141-159.

QUIRINO, Z.G.M. \& MACHADO, I.C. 2001. Biologia da polinização e da reprodução de três espécies de Combretum Loefl. (Combretaceae). Revista Brasileira de Botânica 24:181-193.

RODAL, M.J.N., ANDRADE, K.V.A., SALES, M.F. \& GOMES, A.P.S. 1998. Fitossociologia do componente lenhoso de um refúgio vegetacional no município de Buíque, Pernambuco. Revista Brasileira de Biologia 58:517-526.

SANTOS, M.J.L. 2005. Polinização por beija-flores no Parque Nacional do Catimbau, Nordeste do Brasil. Tese de doutorado, Universidade Federal de Pernambuco, Recife.

SAZIMA, I., BUZATO, S. \& SAZIMA, M. 1995. The Sawbilled Hermit Ramphodon naevius and its flowers in southeastern Brazil. Journal of Ornithology 136:105-206.

SAZIMA, I., BUZATO, S. \& SAZIMA, M. 1996. An assemblage of hummingbird-pollinated flowers in a montane forest in southeastern Brazil. Botanica Acta 109:81-176.

SCHUCHMANN, K.L. 1999. Family Trochilidae (Hummingbirds). In Handbook of the birds of the world (J. del Hoyo, A. Elliott \& J. Sargatal, eds.). Lynx Edicions, Barcelona, p.468-680.

SICK, H. 1997. Ornitologia Brasileira. Nova Fronteira, Rio de Janeiro.

SILVA, J.M.C, SOUZA, M.A., BIEBIER, A.G.D. \& CARLOS, C.J. 2003. Aves da caatinga: status, uso do habitat e sensitividade. In Ecologia e conservação da caatinga (I.R. Leal, M. Tabarelli \& J.M.C. Silva, orgs.). Editora Universitária, Universidade Federal de Pernambuco, Recife, p.237-273.

SIQUEIRA FILHO, J.A. \& MACHADO, I.C. 2001. Biologia reprodutiva de Canistrum aurantiacum E. Morren em remanescente da Floresta Atlântica, Nordeste do Brasil. Acta Botanica Brasilica 15:279-293.
SIQUEIRA FILHO, J.A. \& MACHADO, I.C. 2004. Síndromes de polinização de uma comunidade de Bromeliaceae e biologia floral de Vriesea psittacina (Hooker) Lindley (Bromeliaceae) em Brejo dos Cavalos, Caruaru, Pernambuco. In Brejos de Altitude em Pernambuco e Paraíba-História Natural, Ecologia e Conservação (K.C. Porto, J.J.P. Cabral \& M. Tabarelli, orgs.). Ministério do Meio Ambiente, Brasília, p.227-284.

SNOW, B.K. \& SNOW, D.W. 1972. Feeding niches of hummingbirds in a Trinidad valley. Journal of Animal Ecology 41:471-485.

SNOW, B.K. \& SNOW, D.W. 1980. Relationships between hummingbirds and flowers in Andes of Colombia. Bulletin of the Museum of Natural History (Zoology) 38:105-139.

SNOW, D.W. \& SNOW, B.K. 1986. Feeding ecology of hummingbirds in the Serra do Mar, southeastern Brazil. El Hornero 12:286-296.

SNOW, D.W. \& TEIXEIRA, D.L. 1982. Hummingbirds and their flowers in the coastal mountains of southeastern Brazil. Journal of Ornithology 123:446-450.

STILES, F.G. 1978. Temporal organization of flowering among the hummingbird foodplants of a tropical wet forest. Biotropica 10:194-210.

SUDENE. 1990. Dados pluviométricos do Nordeste - Estado de Pernambuco. Série Pluviométrica 6. Superintendência do Desenvolvimento do Nordeste, Recife.

TEMELES, E.J., LINHART, Y.B., MASONJONES, M. \& MASONJONES, H.D. 2002. The role of flower width in hummingbird bill length-flower length relationships. Biotropica 34:68-80.

TOLEDO, V.M. 1975. La estacionalidad de las flores utilizadas por los colibríes de una selva tropical húmeda en México. Biotropica 7:63-70.

VASCONCELOS, M.F. \& LOMBARDI, J.A. 2000. Espécies vegetais visitadas por beija-flores durante o meio do verão no Parque Estadual da Pedra Azul, Espírito Santo. Melopsittacus 3:36-41.

VASCONCELOS, M.F. \& LOMBARDI, J.A. 2001. Hummingbirds and their flowers in the campos rupestres of Southern Espinhaço Range, Brazil. Melopsittacus 4:3-30.

WOLF, L.L. 1970. The impact of seasonal flowering on the biology of some tropical hummingbirds. The Condor 72:1-14.

WOLF, L., STILES, F. \& HAINSWORTH, F. 1976. Ecological organization of a tropical, highland hummingbird community. Journal of Animal Ecology 32:349-379. 